\title{
Functions and computational principles of serotonergic and related systems at multiple scales
}

\author{
Kae Nakamura ${ }^{1 *}$ and KongFatt Wong-Lin ${ }^{2 *}$ \\ ${ }^{1}$ Department of Physiology, Kansai Medical University, Osaka, Japan \\ 2 Intelligent Systems Research Centre, School of Computing and Intelligent Systems, University of UIster, Northern Ireland, L'Derry, UK \\ *Correspondence: nakamkae@hirakata.kmu.ac.jp; k.wong-lin@ulster.ac.uk
}

Edited by:

Sidney A. Simon, Duke University, USA

Keywords: serotonin 5-HT, neural circuit, computational modeling, dopamine, serotonin, dorsal raphe nucleus, locus coeruleus, pendunculopontine tegmental nucleus

As one of the phylogenetically and ontogenetically oldest neurotransmitters, the monoamine serotonin (5-HT) is derived from tryptophan in neurons within the raphe nuclei, and innervates various parts of the nervous system (Jacobs and Azmitia, 1992). The serotonergic system is complex and can generate multifarious actions (Barnes and Sharp, 1999; Smythies, 2005). There are seven general families of serotonin receptors with multiple receptor subtypes, all of which are $G$ protein-coupled receptors (GPCRs) except one (5-HT3 receptor), which is a ligand-gated ion channel, and these receptors can modulate the release of many major neurotransmitters such as glutamate, GABA, dopamine, acetylcholine, and norepinephrine (Barnes and Sharp, 1999; Smythies, 2005). It can also modulate neuronal excitability and network properties of many targeted brain areas, and regulate mood, cognition and behavior (Smythies, 2005). Dysfunctions of the serotonergic system are implicated in neuropsychiatric disorders including depression and schizophrenia (Müller and Jacobs, 2009). The serotonergic system has been the target of pharmaceuticals for decades, primarily to treat biological and neuropsychiatric disorders. These include antidepressants, antipsychotics, hallucinogens, antimigraine agents, and gastroprokinetic agents (Nichols and Nichols, 2008). Hence, the study of serotonin has high societal impacts.

Although the serotonergic system has been studied for many years, an integrative account of its underlying functions remains elusive. This could be partly attributed to the high variability and heterogeneity in terms of neuronal properties and receptor subtypes, and its extensive connections with other brain regions. Indeed, it has been claimed that serotonin is in involved "in virtually everything, but responsible for nothing" (Jacobs and Fornal, 1995). While there have already been many excellent reviews and books on serotonin and related neural systems (e.g., Jacobs and Azmitia, 1992; Barnes and Sharp, 1999; Smythies, 2005; Müller and Jacobs, 2009), we hope that this collection of recent works provides a complementary and updated coverage of their diverse functions. In particular, unlike previous collections, neurobiologically based computational studies are included in this collection as we consider them to be important toward elucidating some of the underlying principles, especially at the systems level. Hence, we have made a concerted effort to invite both experimental and computational articles in this Research Topic. These works include original results, reviews, and hypothesis over multiple levels: from receptors and channels, to neuronal circuits and finally to behavior and neuropsychiatric disorders.

At the receptor and cellular levels, Maejima et al. (2013) discussed various GPCRs and ion channels in the serotonin regulation and introduced optogenetic techniques that modulate intracellular signaling to more finely control the serotonergic systems for studies of their functions. The activation of the serotonin receptors was determined by its release and uptake dynamics. Unlike other more commonly studied neurotransmitters such as acetylcholine for example, the release and uptake dynamics of serotonin is not well characterized. Dankowski and Wightman (2013) reviewed the challenges and developments of fast-scan cyclic voltammetry to monitor serotonin at the subsecond (maybe millisecond) timescale in both in vitro and in vivo conditions.

At the neuronal circuit level, Celada et al., 2013 provided a comprehensive review on cortical modulation of serotonin. In particular, the prefrontal cortex, linked to executive brain functions, seemed to form closed-loop interactions with the serotonin neurons in the dorsal raphe nucleus. This review was well complemented by biologically realistic computational modeling works of serotonin modulation on the prefrontal cortex. In Wang and Wong-Lin (2013), a biologically motivated model was developed to investigate how the co-modulation of serotonin and dopamine in the prefrontal cortex could result in complex, non-intuitive neuronal circuit dynamics, thus challenging current simpler theories on neuromodulation. Cano-Colino et al. (2013) incorporated serotonin modulation into an established computational model of the prefrontal cortex performing spatial working memory tasks. The model showed that excessive serotonin could impede task performance, and interestingly predicted that serotonin levels could affect neuronal memory fields.

Besides the cortex, serotonin is also known to modulate important subcortical brain regions. Using a mathematical model of multiple brain regions, Reed et al. (2013) demonstrated the potential roles of serotonin in maintaining homeostasis in the basal ganglia (via the frontal cortex) under dopamine depletion (e.g., in Parkinson's disease). In Nakamura (2013), the neural circuit architecture of the dorsal raphe nucleus and other key subcortical brain regions involved in reward-based decision making and learning were discussed with emphasis on the neural circuit. The dorsal raphe nucleus has strong anatomical and functional connectivity with neighboring structures including the pendunculopontine tegmental nucleus (PPTg) and the 
locus coeruleus (LC), where many acetylcholine and noradrenergic neurons are found, respectively, (Koyama and Koyama, 1993; Martinez-Gonzalez et al., 2011). Indeed, Okada and Kobayashi (2013) showed that PPTg neurons exhibit similar tracking of future reward expectation as neurons in the dorsal raphe nucleus. Tsuruoka et al. (2012) reviewed the role of LC on pain control, which might be involved in aversive information processing.

It has been proposed that reinforcement learning models can be used as a platform for studying neurological and neuropsychiatric disorders (Maia and Frank, 2011). In this collection, Herzallah et al. (2013) dissociated among depressed patients with and without antidepressant medication, and healthy control subjects by observing the performance in learning from positive (reward) and negative (punishment) feedback. Castro-Rodrigues and Oliveira-Maia (2013) provided a useful commentary on this important original work. Finally, the comprehensive review by Asher et al. (2013) proposed a closed-loop paradigm toward understanding serotonergic roles in decision making by involving behavioral experiments, game theory, computational modeling, and human-robotic interaction, a truly integrative neuroscience approach.

We hope that this issue will provide a comprehensive review of the diverse and complex functions and computations of serotonergic and related systems at multiple scales of investigation. We wish that this will motivate and inspire a more integrative research approach from cellular to systems level toward understanding neuromodulatory systems.

\section{ACKNOWLEDGMENTS}

We would like to thank all the authors for participating as well as help from the Frontiers Neuroscience Editorial Office staff and encouragement from the chief editor, Sid Simon. We would also wish to thank Sid Simon for comments on this editorial, and the reviewers, whose contributions significantly helped to improve the published papers that constituted this Research Topic.

\section{REFERENCES}

Asher, D. E., Craig, A. B., Zaldivar, A., Brewer, A. A., and Krichmar, J. L. (2013). A dynamic, embodied paradigm to investigate the role of serotonin in decisionmaking. Front. Integr. Neurosci. 7:78. doi: 10.3389/fnint.2013.00078

Barnes, N. M., and Sharp, T. (1999). A review of central 5-HT receptor and their function. Neuropharmacology 38, 1083-1152. doi: 10.1016/S00283908(99)00010-6

Cano-Colino, M., Almeida, R., and Compte, A. (2013). Serotonergic modulation of spatial working memory: predictions from a computational network model. Front. Integr. Neurosci. 7:71. doi: 10.3389/fnint.2013.00071

Castro-Rodrigues, P., and Oliveira-Maia, A. J. (2013). Exploring the effects of depression and treatment of depression in reinforcement learning. Front. Integr. Neurosci. 7:72. doi: 10.3389/fnint.2013.00072

Celada, P., Puig, M. V., and Artigas, F. (2013). Serotonin modulation of cortical neurons and networks. Front. Integr. Neurosci. 7:25. doi: 10.3389/fnint.2013.00025
Dankowski, E. C., and Wightman, R. M. (2013). Monitoring serotonin signaling on a subsecond time scale. Front. Integr. Neurosci. 7:44. doi: 10.3389/fnint.2013.00044

Herzallah, M. M., Moustafa, A. A., Natsheh, J. Y., Abdellatif, S. M., Taha, M. B., Tayem, Y. I., et al. (2013). Learning from negative feedback in patients with major depressive disorder is attenuated by SSRI antidepressants. Front. Integr. Neurosci. 7:67. doi: 10.3389/fnint.2013.00067

Jacobs, B. L., and Azmitia, E. C. (1992). Structure and function of the brain serotonin system. Physiol. Rev. 72, 165-229.

Jacobs, B. L., and Fornal, C. A. (1995). "Serotonin and behaviour: a general hypothesis," in Psychopharmacology: The Fourth Generation of Progress, eds F. E. Bloom and D. J. Kupfer (New York, NY: Raven), 461-470.

Koyama, Y., and Koyama, Y. (1993). Mutual interactions among cholinergic, noradrenergic and serotonergic neurons studied by ionophresis of these transmitters in rat brainstem nuclei. Neuroscience 55, 1117-1126. doi: 10.1016/03064522(93)90325-A

Maejima, T., Masseck, O. A., Mark, M. D., and Herlitze, S. (2013). Modulation of firing and synaptic transmission of serotonergic neurons by intrinsic $G$ protein-coupled receptors and ion channels. Front. Integr. Neurosci. 7:40. doi: 10.3389/fnint.2013.00040

Maia, T. V., and Frank, M. J. (2011). From reinforcement learning models to psychiatric and neurological disorders. Nat. Neurosci. 14, 154-162. doi: $10.1038 / \mathrm{nn} .2723$

Martinez-Gonzalez, C., Bolam, J. P., and Mena-Segovia, J. (2011). Topographical organization of the pendunculopontine nucleus. Front. Neuroanat. 5:22. doi: 10.3389/fnana.2011.00022

Müller, C. P., and Jacobs, B. L. (2009). Handbook of the Behavioral Neurobiology of Serotonin. London: Academic Press.

Nakamura, K. (2013). The role of the dorsal raphe nucleus in reward-seeking behavior. Front. Integr. Neurosci. 7:60. doi: 10.3389/fnint.2013.00060

Nichols, D. E., and Nichols, C. D.(2008). Serotonin receptors. Chem. Rev. 108, 1614-1641. doi: 10.1021/cr078224o

Okada, K., and Kobayashi, Y. (2013). Reward prediction-related increases and decreases in tonic neuronal activity of the pedunculopontine tegmental nucleus. Front. Integr. Neurosci. 7:36. doi: 10.3389/fnint.2013.00036

Reed, M. C., Nijhout, H. F., and Best, J. (2013). Computational studies of the role of serotonin in the basal ganglia. Front. Integr. Neurosci. 7:41. doi: 10.3389/fnint.2013.00041

Smythies, J. (2005). Section V. Serotonin system. Int. Rev. Neurobiol. 64, 217-268. doi: 10.1016/S0074-7742(05)64005-6

Tsuruoka, M., Tamaki, J., Maeda, M., Hayashi, B., and Inoue, T. (2012). Biological implications of coeruleospinal inhibition of nociceptive processing in the spinal cord. Front. Integr. Neurosci. 6:87. doi: 10.3389/fnint.2012.00087

Wang, D.-H., and Wong-Lin, K. (2013). Comodulation of dopamine and serotonin on prefrontal cortical rhythms: a theoretical study. Front. Integr. Neurosci. 7:54. doi: 10.3389/fnint.2013.00054

Received: 18 February 2014; accepted: 19 February 2014; published online: 07 March 2014.

Citation: Nakamura K and Wong-Lin K (2014) Functions and computational principles of serotonergic and related systems at multiple scales. Front. Integr. Neurosci. 8:23. doi: $10.3389 /$ fnint.2014.00023

This article was submitted to the journal Frontiers in Integrative Neuroscience.

Copyright (c) 2014 Nakamura and Wong-Lin. This is an open-access article distributed under the terms of the Creative Commons Attribution License (CC BY). The use, distribution or reproduction in other forums is permitted, provided the original author(s) or licensor are credited and that the original publication in this journal is cited, in accordance with accepted academic practice. No use, distribution or reproduction is permitted which does not comply with these terms. 